\title{
DESCRIBING AND MEASURING THE ENGINEERING KNOWLEDGE BASED USING CONCEPT DOMAINS
}

\author{
Jason Grove ${ }^{1}$, Marios Ioannidis ${ }^{1}$, and Derek Wright ${ }^{2}$ \\ University of Waterloo, ${ }^{1}$ Department of Chemical Engineering, ${ }^{2}$ Department of Electrical and Computer Engineering \\ jagrove@uwaterloo.ca
}

\begin{abstract}
We describe the chemical engineering knowledge base in terms of five distinct concept domains: i) mathematics and computation, ii) conservation, iii) equilibrium and spontaneity, $i v)$ rates, and v) the structure and property of materials. These concept domains underpin the curriculum and evolve from disparate subject domains presented in the first year into a cohesive whole by graduation. The knowledge base for chemical engineering can thus be expressed in terms of achieving threshold concepts related to each of these domains.

This formulation of the knowledge base suggests that it may be examined using concept inventory testing. We provide examples of how such testing can be implemented in order to produce meaningful data on students' level of concept attainment. We believe that this approach may be of interest to others as a robust and sustainable method for the ongoing assessment of $C E A B$ Graduate Attribute 1.
\end{abstract}

Keywords: concept inventory; graduate attributes; knowledge base; threshold concept

\section{INTRODUCTION}

The Canadian Engineering Accreditation Board (CEAB) specifies 12 Graduate Attributes that must be defined and assessed as part of a continual improvement process for the academic program [1]. The first attribute is defined as: A knowledge base for engineering: Demonstrated competence in university level mathematics, natural sciences, engineering fundamentals, and specialized engineering knowledge appropriate to the program. In order to measure this Graduate Attribute, it is necessary to formulate an operational definition of what constitutes "competence... appropriate to the program". In this paper, we describe our process for developing this definition and its subsequent organization into five conceptual domains. Competence in the discipline (chemical engineering) is then defined in terms of achieving, internalizing and then integrating the understanding of threshold concepts in these five domains. A rich literature exists on the threshold concepts and we do not seek to reproduce it here; for a recent review, see [2].

\section{CONCEPT DOMAIN MAPPING}

\subsection{Process for the Identification of Concept Domains}

Following a curriculum mapping exercise facilitated by the University of Waterloo's Centre for Teaching Excellence, the chemical engineering program developed a detailed curriculum map. While rich in information, this map was somewhat unwieldy in terms of its high-level organization and the authors began an informal discussion around how it should be organized. The initial direction was towards organizing the learning outcomes in thematic areas associated with different specialties of chemical engineering. However, this was unsatisfying due to i) the overlap between different areas, ii) the large number of specialties in chemical engineering, and, iii) the overall lack of insight provided by this approach. Simultaneously, we were discussing the difference between the knowledge base and problem analysis graduate attributes. These two attributes are perhaps the most readily measurable thanks to the wealth of assignment and examination questions that require their application; however, these data are often confounded in the sense that both attributes are being tested simultaneously and therefore our discussions often focused on how the two attributes could be meaningfully differentiated in terms of both intent and measurement.

The result of this process was a recognition that the knowledge base can be expressed in terms of understanding in a limited number of concept domains. Competence in the knowledge base for chemical engineering can then be thought of as passing a set of threshold concepts within these domains. Assessment can be achieved via concept testing (vide infra).

\subsection{Concept Domain Mapping}

We identified the following five concept domains for chemical engineering:

1. Conservation. "Stuff" does not magically appear or disappear (the stuff may be mass, energy, momentum, etc.). 
2. Equilibrium and spontaneity. Physical systems may reach situations of equilibrium where no net changes (movement or transformation of "stuff") take place.

3. Rates of reaction and transport. It takes time for "stuff" to move or transform.

4. Structure and properties of materials. Materials have properties that may be described in terms of a hierarchical structure and interactions.

5. Mathematics and computation. Relationships between physical quantities in all of the above can be described and manipulated both symbolically and numerically using a common precise language

We believe that this organization of the chemical engineering knowledge base is helpful in providing a framework against which to measure student understanding. With this in mind, we undertook an exercise to map each of our core courses to its associated conceptual domains. The results are shown in Figure 1.

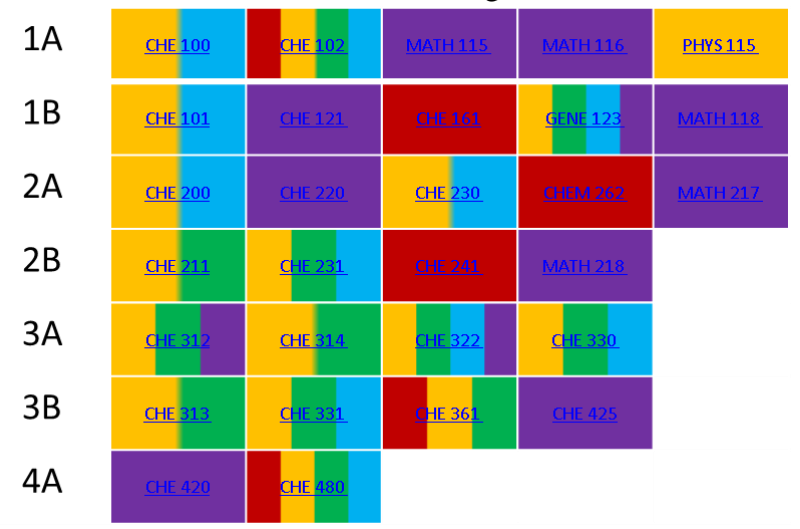

Fig. 1. Curriculum mapping of core technical courses by term (left column) into five concept domains:

yellow-conservation; green-rates; blue-equilibrium; red-materials; purple-mathematics.

A number of interesting observations can be drawn. First, the exercise turned out to be extremely easy and rapid, lending us some reassurance that the conceptual framework is a rational one. Second, we observe that the number of conceptual domains associated with each course tends to increase each term. The exceptions to this are: i) two first-year courses that are common to many different engineering disciplines and have characteristics of survey courses in those areas, and, ii) courses in mathematics. This observation reflects the organization of the curriculum, whereby earlier courses are introductory and so naturally confine themselves to one or two conceptual domains, while later courses reflect more advanced and practical material that requires the integration of all concept domains.

\subsection{Application to Other Engineering Disciplines}

We would like to note the generality of the concept domains identified above and suggest that these general categories may be applied to other engineering disciplines, with variation in the details of what is included within each domain. For examples, in chemical engineering, the most important equilibrium concepts all concern dynamic equilibria, with static equilibrium being treated only briefly (perhaps in regard to fluids). In civil engineering static equilibria would presumably feature much more prominently. Similarly, chemical engineers need only a basic appreciation of conservation of charge, while electrical engineers would need a much conceptual understanding in this area.

\section{CONCEPT TESTING}

Concept tests (or concept inventories) are typically a set of conceptual multiple-choice problems with carefully designed distractors intended to identify common misunderstandings. Data from such questions can provide clear and meaningful measurements of what students do and do not understand. The main challenge is in developing and validating appropriate questions. The approach is welldocumented approach and there are a number of published and validated inventories available.

We have blended questions from these published questions with others that have been written in-house, that also include other short-answer formats such as graph sketching. The results have been quite illuminating and we believe will be powerful in terms of providing meaningful evidence for curriculum improvement. We provide a number of illustrative examples in the following sections.

\subsection{Examples}

\subsection{1. "Clean" Measurement of Knowledge Base}

The first example illustrates how concept testing can disentangle the knowledge base from problem analysis in measurement. The introductory Chemical Engineering Concepts course has a significant focus on performing material balances around chemical processes. Appendix A. 1 shows a conceptual question and standard long-answer examination question used in this course. Note that the long-answer question involved sketching precisely the process flow diagram provided in the conceptual question and was largely intended to address student understanding of the purpose of a recycle stream with purge. The average score on the standard examination question was $84 \%$, suggesting that students have a good understanding of this concept, based on an assessment of their ability to solve this numerical problem. However, only $21 \%$ of the same students were able to correctly answer the related conceptual question. From this, we conclude that student understanding of the purpose of the recycle purge is in fact quite poor; despite this, they are sufficiently familiar with a problem analysis technique that will allow them to correctly deduce the value of all the unknowns in the system. This illustrates the value of attempting to 
disentangle the problem analysis from the underlying conceptual basis of the problem.

\subsubsection{Robust Class-Level Comparison}

The second example illustrates how concept testing data can be used to enable robust comparisons between classes. Three concept problems related to material balances developed and published (questions 10,11 and 18) by Shalcross [3] were used as part of the final exam for the same Chemical Engineering Concepts class as in the previous example. Since Shalcross provided details of the class performance, it is possible to compare the performance of our class in these problems directly to the class at the University of Melbourne. In this case, performance was very similar with Waterloo (Melbourne) students answering these three questions correctly $79.5 \%$ (76.5\%), $70.5 \%(69.1 \%)$ and $53.4 \%(51.5 \%)$ of the time. Please note that these data are provided for illustration and we do not propose that this very limited sample provides for a meaningful comparison; however we do contend that a carefully designed test would provide a basis for a meaningful comparison either between institutions or over time at the same institution (either following a cohort longitudinally or comparing different classes at the same point in the program). For the longitudinal case, we also note that repeated use of identical or very similar questions provides opportunities to identify when misconceptions are corrected (or new ones arise).

\subsubsection{Graphing Question Example}

Our final example illustrates a different question type not usually included in the concept inventory literature but that that we have found to be informative. We have developed a number of questions in which students are asked to sketch graphs rather than selecting from multiple choice options. Although it might be possible to develop a set of graphs to provide as multiple-choice options, we have found that the effort in grading is very slight since it is immediately apparent whether the results are right or wrong. An example of such a problem is provided in Appendix A.2.

\subsection{Use of Concept Tests for Program Assessment}

In order for concept testing to provide meaningful data to inform program improvement, considerable effort and planning is required. The preparation of concept inventory questions can be very time-intensive (although we do note that having developed one question it is sometimes possible to spin off a number of variations quite rapidly by making small adjustments). On the other hand, the problems are multiple choice and can relatively easily be memorized and reproduced with at least enough accuracy to enable the correct answer to be identified. Students at our institution have previously shown themselves to be adept at assembling collections of final examination questions, even when they are not released. Furthermore, given that our objective was to test fundamental conceptual understanding at the program level, it is not clear that deploying concept questions at the same time as final examinations is appropriate, since it may be better to do so away from examination cramming and in a more holistic manner in terms of covering all five concept domains. We are therefore beginning to implement a regular termly concept test during a class seminar hour; to encourage participation, students are rewarded with a $1 \%$ bonus in their laboratory course for attempting the test and a further $1 \%$ for scoring a minimum of half marks. While the questions cannot be released back to the students (many of the published concept inventories require an undertaking not to do so, as a result of the considerable effort required to prepare the questions in the first place), we are working on a mechanism to communicate some information on their performance back to students, probably in the form of a score categorized in the five identified concept domains.

Finally, we note that students are generally unfamiliar with concept testing-type questions. In particular, they may not immediately recognize the necessarily precise use of language in many concept questions; for this reason, it is beneficial to highlight the importance of careful reading to the students and to consider providing at least one or two practice problems.

\section{CONCLUSION}

The organization of the engineering knowledge base into a small number of conceptual domains and their assessment via concept testing may provide a powerful and efficient mechanism for the assessment of this attribute.

\section{References}

[1] Engineers Canada Accreditation Board, 2015 Accreditation Criteria and Procedures, 2015, \{ISSN: 1708-8054\}.

[2] S. Barradell, "The identification of threshold concepts: A review of theoretical complexities and methodological challenges," Higher Education, vol. 65, no. 2, pp. 265-276, 2013.

[3] D. C. Shallcross, "A concept inventory for material and energy balances," Education for Chemical Engineers, vol. 5, no. 1, e1-e12, 2010.

\section{APPENDIX A:}

\section{A.1 Example Conceptual and Traditional Problems}

\section{Conceptual problem formulation:}

Consider the process flow diagram shown below. The inlet stream consists of the reactants plus 5\% inerts. The single-pass conversion of the reactor is known to be less than $50 \%$. The reactor products are separated into a pure product stream and a recycle stream. A fraction $\alpha$ of the 
recycle stream is purged and the remainder recycled to the reactor inlet.

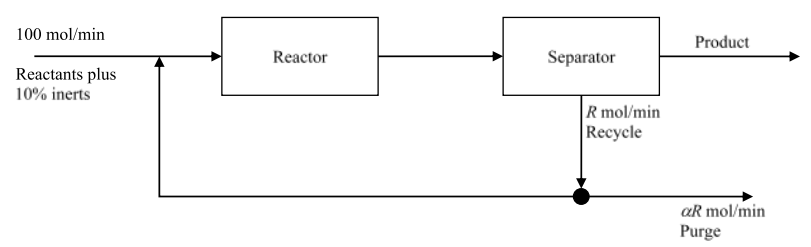

Consider the flowrate of the purge and recycle streams. Indicate clearly with an $\mathrm{X}$ which combinations of recycle rate $R$ and purge fraction $\alpha$ may give valid solutions.

\section{Traditional problem formulation:}

Production of phtalic acid anhydride $\left(\mathrm{C}_{8} \mathrm{H}_{4} \mathrm{O}_{3}\right)$ is obtained by the oxidation of $o$-xylene:

$$
\mathrm{C}_{8} \mathrm{H}_{10}+3 \mathrm{O}_{2} \rightarrow \mathrm{C}_{8} \mathrm{H}_{4} \mathrm{O}_{3}+3 \mathrm{H}_{2} \mathrm{O}
$$

The fresh feed contains 6 moles o-xylene/hour and 94 moles air/hour. It is combined with a recycle stream and enters the reactor. The reactor output, 118 moles/hour, is sent to a condenser where all of the $\mathrm{C}_{8} \mathrm{H}_{4} \mathrm{O}_{3}$ produced is removed as the product stream (pure $\mathrm{C}_{8} \mathrm{H}_{4} \mathrm{O}_{3}$ stream). The unreacted $\mathrm{C}_{8} \mathrm{H}_{10}, \mathrm{O}_{2}, \mathrm{H}_{2} \mathrm{O}$ and nitrogen are recycled. A purge stream is required to prevent build-up of nitrogen in the system. The purge gas is $78 \%$ nitrogen.

a. [3 pts] Draw a diagram and label known/unknown variables.

b. [4 pts] Perform a degree of freedom analysis for each of the reactor, the condenser and the overall process. c. [14 pts] Estimate, purge flow rate $(\mathrm{mol} / \mathrm{h})$, product flow rate $(\mathrm{mol} / \mathrm{h}$ ) and the purge composition ( $\mathrm{mol} \%$ of each species).

d. [4 pts] Estimate the recycle flow rate $(\mathrm{mol} / \mathrm{h})$, that is the flow rate of the stream after the purge returning to the reactor.

Hint: you will notice from the stoichiometry of the reaction that the total number of moles does not change after the reaction.

Note that a correct solution to this traditional problem requires the students to correctly sketch and label a process flow diagram identical to the one provided in the conceptual question above.

\section{A.2 Example Graphing Question}

Imagine a round-bottom flask (sketched below) filling with water. Sketch a graph of the height of water in the flask as a function of the volume of water in the flask.

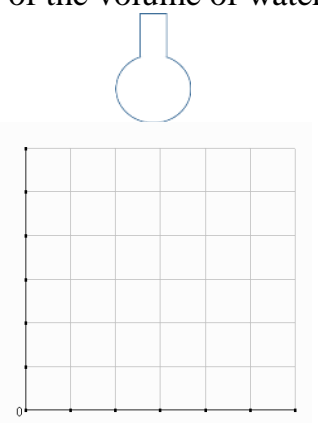

